\title{
Tetralogy of Fallot- Not an Innocuous Abnormality in Adult
}

\author{
Ayush Dubey ${ }^{1}$, Nipun Bawiskar², Sunil Kumar³, Swapnil Lahole ${ }^{4}$, Ayush Somani ${ }^{5}$
}

\begin{abstract}
${ }^{1}$ Department of Medicine, Jawaharlal Nehru Medical College, Datta Meghe Institute of Medical Sciences (Deemed to be University), Wardha, Maharashtra, India. ${ }^{2}$ Department of Medicine, Jawaharlal Nehru Medical College, Datta Meghe Institute of Medical Sciences (Deemed to be University), Wardha, Maharashtra, India. ${ }^{3}$ Department of Medicine, Jawaharlal Nehru Medical College, Datta Meghe Institute of Medical Sciences (Deemed to be University), Wardha, Maharashtra, India. ${ }^{4}$ Department of Medicine, Jawaharlal Nehru Medical College, Datta Meghe Institute of Medical Sciences (Deemed to be University), Wardha, Maharashtra, India. ${ }^{5}$ Department of Medicine, Jawaharlal Nehru Medical College, Datta Meghe Institute of Medical Sciences (Deemed to be University), Wardha, Maharashtra, India.
\end{abstract}

\section{INTRODUCTION}

Among cyanotic congenital heart disease, Fallot's tetralogy most commonly presents in childhood and rarely goes beyond second decade as death is inevitable without surgical correction of the abnormality. Here we report about unusual course of an uncorrected tetralogy, in which patient reach adulthood and presented only with seizure later found to have brain abscess.

Fallot's tetralogy (TOF) accounts for $10 \%$ of cyanotic congenital heart disease, which has combinations of a) ventricular septal defect, b) overriding aorta, c) right ventricular hypertrophy, and d) right ventricular outflow tract obstruction.[1] Although known complication, cerebral abscess has been reported in patients with CHD mostly at the age of 4-7 years, only rarely in adults as their survival is minimal without surgical interventions.[2] In this case, a 49 year old female who presented with a cerebral abscess later diagnosed as TOF on $2 \mathrm{D}$ echocardiography.

\section{PRESENTATION OF CASE}

A tribal female aged 49 year with rural background presented in medicine emergency unit with high grade fever, headache, generalised seizure of and on since 5 days. She was also having breathlessness since one week. History of any chronic illness like pulmonary tuberculosis, bronchial asthma, ischemic heart disease, diabetes mellitus and hypertension in past were absent. She was non-alcoholic, non-smoker, and strictly denied any use of medications like corticosteroids or ayurvedic drugs.

On examination, the patient was drowsy due to post ictal stage and febrile. Her pulse was regular with 106 rate per min and blood pressure recorded in right arm supine position was $130 / 80 \mathrm{~mm}$ of $\mathrm{Hg}$. Signs of meningism were present in the form of positive neck stiffness and Kernig's sign. . She was pale with grade 2 clubbing in both the hands. Her precordium were hyperdynamic with pansystolic murmur all over it. Other systemic examination was normal. On laboratory investigations, her haemoglobin was $7.8 \mathrm{gm} / \mathrm{dl}$ and the white blood cell count was $16,800 \mathrm{c} / \mathrm{mm}$. Liver and kidney function tests were normal. Routine examination of Urine was normal.

Her ESR was $12 \mathrm{~mm}$ in first hour. Chest X-Ray showed no obvious abnormality. Blood cultures from three different sites one hour apart were sent in view of fever and cardiac finding which showed no growth. In view of cardiovascular examination 2D echocardiography was done which was suggestive of congenital heart disease having large misaligned Ventricular Septal Defect with overriding of aorta, and severe valvular and subvalvular pulmonary stenosis. Right Atria and Ventricle were dilated. There was no vegetation. All the features were suggestive of Tetralogy of Fallot shown in figure 1.
Corresponding Author:

Sunil Kumar,

Professor and HOD,

Department of Medicine,

Jawaharlal Nehru Medical College, Datta Meghe Institute of Medical Sciences (Deemed to be University), Wardha, Maharashtra, India.

E-mail: sunilkumarmed@gmail.com

\section{DOI: $10.14260 / j e m d s / 2020 / 304$}

Financial or Other Competing Interests: None.

How to Cite This Article:

Dubey A, Bawiskar N, Kumar S, et al. Tetralogy of fallot- not an innocuous abnormality in adult. J. Evolution Med. Dent. Sci. 2020;9(16):1397-1398, DOI: 10.14260/jemds/2020/304

Submission 01-02-2020,

Peer Review 05-04-2020,

Acceptance 11-04-2020,

Published 20-04-2020.

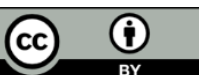




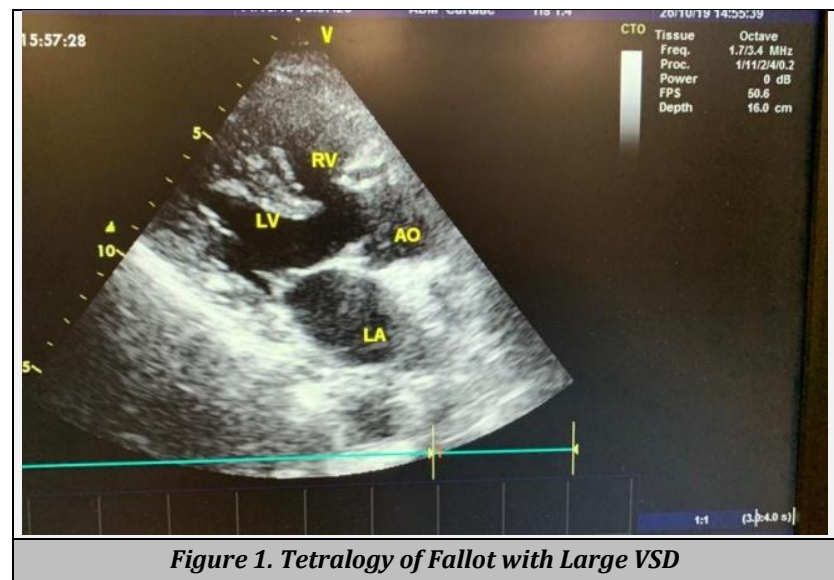

In view of headache and seizure she had undergone computerized tomography scan of brain which revealed well defined round to oval ring enhancing lesion in left high parietal region with surrounding perilesional oedema most likely suggestive of Cerebral abscess. [figure 2] Neurosurgical opinion was taken, and cerebral abscess was drained out with all precaution and high-risk consent. Though culture of the pus was negative, patient was kept on higher antibiotic as injectable vancomycin, in combination with ceftriaxone and metronidazole for 21 days. She was also put on antiepileptic drugs as levetiracetam $500 \mathrm{mg}$ twice a day. Patient responded well with no focal deficits on neurological examination. She was referred to cardiothoracic surgeons for following up of the tetralogy of Fallot, but due to financial constraint surgical correction were deferred. Patient was put on conservative management.

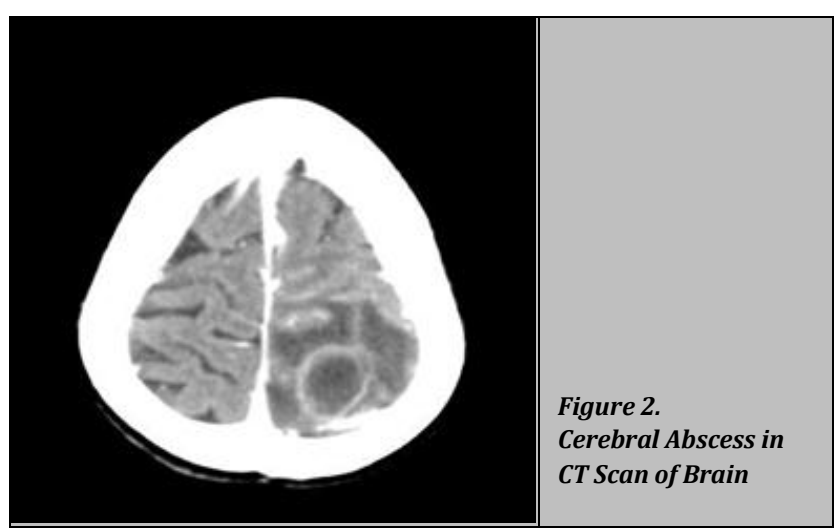

DISCUSSION

TOF is one of the most common forms of cyanotic congenital heart disease first described by Arthur Fallot in year 1888. There is evidence that only $2 \%$ of all such patients reach the fourth decade of life without surgical corrections. Possible hypothesis may be large VSD, functional left ventricle and a mild sub-pulmonary stenosis (which does not lead to severe hypoxia), and to protect the pulmonary vasculature. Our patient survived 49 years of age and still counting due to mild pulmonary hypertension and large VSD. Brain abscess is a serious and fatal complication seen in children, but only few cases had been reported in an adult having TOF.[1,2,3,4] Our case probably rarest in this part of country survived up to 49 years of age without any serious complication before presented with brain abscess. Possible mechanism may be hyperviscosity, which leads to small and large infarct due to decreased flow in the microcirculation areas and infection in shunted blood results in focal cerebritis, thence abscess formation. Sometimes hypoxia can lead to abscess formation, though mechanism is not clear.[4] The most common sites for brain abscess are parietal, frontal and temporal lobes of the brain. ${ }^{[4,5]}$ In our patient the site was left high parietal region. Clinically, the patients usually present with vomiting, fever, headache, seizures, focal neurologic signs in younger age.[6,3] In our case the patient presented for the first time at the age of 49 years with seizure as an underlying manifestation of brain abscess. We used ceftriaxone and metronidazole in combination with vancomycin for the treatment of brain abscess considering various spectrum. With regards to surgical interventions, brain abscess may be removed through osteoplastic craniotomy with removal of abscess capsule, or the abscesses may be treated by aspiration and drainage of the abscess under stereotaxic guidance as we had done in our case. $[2,3]$

\section{CONCLUSIONS}

In any case of congenital heart disease if the patient is presenting with some sort of neurological deficit we should always keep possibility of brain abscess in mind while treating this patient. Imaging brain plays a vital role in diagnosing it and hence should be performed in every patient of CHD with neurological deficit.

\section{REFERENCES}

[1] Sousa P, Santos W, Marques N, et al. A 72-year-old woman with an uncorrected tetralogy of Fallot presenting with possible pulmonary endocarditis: a case report. Journal of Medical Case Reports 2013;7:150.

[2] Lumbiganon P, Chaikitpinyo A. Antibiotics for brain abscesses in people with cyanotic congenital heart disease. Cochrane Database Syst Rev 2013;(3):CD004469.

[3] Sung CW, Jung JH, Lee SH, et al. Brain abscess in an adult with atrial septal defect. Clinical Cardiology 2010;33(4):E51-E3.

[4] Kumar K. Neurological complications of congenital heart disease. Indian J Pediatr 2000;67(4):287-91.

[5] Sani MU, Mukhtar-Yola M, Karaye KM. Spectrum of congenital heart disease in a tropical environment: an echocardiography study. J Natl Med Assoc 2007;99(6):665-9.

[6] Chandrasekaran B, Wilde P, McCrea WA. Tetralogy of Fallot in a 78-year-old man. $\mathrm{N}$ Engl J Med 2007;357(11):1160-1. 